\title{
The evolution of senescence under curtailed life span in laboratory populations of Musca domestica (the housefly)
}

\author{
DAVID H. REED* \& EDWIN H. BRYANT \\ Department of Biology, University of Houston, Houston, TX 77204-5513, U.S.A.
}

\begin{abstract}
The evolution of senescence may be explained by two different, but not mutually exclusive, genetic mechanisms. The antagonistic pleiotropy hypothesis predicts that senescence is a consequence of the fixation of alleles with pleiotropic effects favouring early life fitness, but bearing a cost in later life. The mutation-accumulation hypothesis attributes senescence to the accumulation of deleterious mutations with late-acting effects on fitness in mutation-selection balance. Experiments were carried out on the housefly, Musca domestica, in which large and small populations were maintained so that reproduction was limited to four or five days after reaching sexual maturity. Longevity declined significantly under the husbandry protocol and was largely the same in large and small populations; this is consistent with the random accumulation of deleterious alleles affecting longevity under curtailed life span, although laboratory adaptation cannot be ruled out entirely as a causal mechanism. An analysis of life-history data did not provide evidence for a trade-off between longevity and age at sexual maturity, developmental time, or dry body weight, but there was an apparent tradeoff between longevity and early progeny production, in support of antagonistic pleiotropy.
\end{abstract}

Keywords: housefly, life-history, senescence.

\section{Introduction}

An individual's risk of death due to accident, disease, malnutrition, or predation is cumulative over time, so that an allele that affects fitness early in life has an equal or greater consequence than one that affects fitness later (Williams, 1957; Hamilton, 1966; Rose, 1991). The relative indifference of natural selection to alleles that reduce biological performance in later life has given rise to the concept of senescence, which manifests itself as a decline in fertility and an increase in mortality with advancing age (Medawar, 1952; Rose, 1991).

Two major evolutionary hypotheses have been proposed to explain senescence. The antagonistic pleiotropy hypothesis attributes senescence to the fixation of alleles with pleiotropic effects that favour early life fitness but bear a cost in later life (Williams, 1957; Rose, 1991). In contrast, the mutation accumulation hypothesis attributes senescence to the accumulation of deleterious mutations with late-acting effects on fitness (Medawar, 1952). It has been particularly difficult to disentangle the two causes of senescence in experimental studies, and it is likely that both play a vital role in determining

*Correspondence. E-mail: judodoc@hotmail.com/ebryant@uh.edu ultimate longevity (see Partridge \& Barton, 1993; Charlesworth, 1994; Promislow \& Tartar, 1998; for recent reviews).

Edney \& Gill (1968) proposed a straightforward experiment of curtailing life span to test for the effects of mutation accumulation on longevity. Under curtailed life span late-acting deleterious mutations are not exposed to selection, and thereby are allowed to accumulate freely, resulting in shortened longevity when total life span is assayed. While such experiments on curtailing life span do not exclude the effects of antogonistic pleiotropy, they can provide evidence in favour of mutation accumulation (Promislow \& Tartar, 1998). Experimental populations of Drosophila, Tribolium and the bean weevil, Acanthoscelides obtecus, kept under a shortened generation regime, have shown such decreased longevity (Sokal, 1970; Rose, 1984; Service et al., 1985; Mueller, 1987; Partridge \& Fowler, 1992; Tucic et al., 1997).

Most of the data on the evolution of longevity have used Drosophila (see reviews by Rose, 1991; Partridge \& Barton, 1993; Promislow \& Tartar, 1998), and there is a continuing need to evaluate changes in longevity in other species. This paper provides such additional data by comparing longevities in populations of the housefly, 
Musca domestica L., in which life span was severely curtailed for 24 generations. Because deleterious mutations should accumulate independently among such experimental lines, we utilize crosses among lines to provide additional evidence in favour of mutation accumulation (Mueller, 1987). Finally, discrete-generation protocols have been used to maintain most laboratory stocks in Drosophila, including those for initiating many longevity experiments (Pletcher et al., 1999), and so it is important to determine, independent of tests of the causes of senescence, how fast longevity may decline under curtailed life span. By starting with a newly established strain of the housefly we also provide an estimate of the rate of change in longevity resulting from the discrete-generation protocol.

\section{Materials and methods}

\section{Controls}

In any experiment on longevity, control lines are problematic, because all lines are usually kept under some form of discrete generation management protocol, including our base population, that would alter longevity over time in any base population (Promislow \& Tartar, 1998). Thus, resampling a large base population over time would not provide an adequate control. Even if this were not the case, a large control population would most likely accumulate beneficial mutations over the course of an experiment, particularly if laboratory adaptation is occurring, that would partially mask the effects of mutation accumulation (Lynch et al., 1999). Cryopreserved lines are perhaps the only ideal control but in practice are not without technical problems (Pletcher et al., 1999), and such techniques are not available for the housefly, which is extremely sensitive to low temperature (Rosales et al., 1994).

One possible approach to the dilemma of a control is to compare the effects of mutation accumulation on experimental lines of different sizes, as suggested by Lynch et al. (1999). To the extent curtailed life span renders late-acting mutations neutral they should accumulate independently of population size (Kimura, 1983) and longevity should decrease uniformly across populations of different size. If, on the other hand, selection was involved, either acting directly on late-acting mutations or indirectly through pleiotropic effects across the life span, large populations should exhibit increased late-life mortality relative to smaller populations. In this paper, we apply curtailed life span to housefly populations of both large and small size and compare changes in longevity over time. In addition, crosses among the lines to restore ancestral longevity were used to infer independent accumulation of muta- tional effects across line, in the manner of Mueller (1987). By evaluating such crosses simultaneously with experimental lines, they also provide a control for systematic environmental effects.

\section{Experimental protocol}

The experiment was initiated with 141 female flies and an excess of males (to ensure insemination of all females) captured from a wild population in Houston, TX. Eggs were collected in mass from these wild-caught flies, and the population was expanded to $>4000$ flies within several generations. After five generations to allow for adaptation to the laboratory environment treatment, lines were initiated from the BASE population (= generation 0 of the experiment): (i) four lines, designated LARGE, were initiated and maintained for each generation, with a random sample of eggs sufficient to produce a minimum of 1000 adults; (ii) twelve lines, designated SMALL, were initiated and maintained, with a random sample of eggs sufficient to produce approximately 130 adults. In each generation, flies were counted to determine the exact number of adults, yielding a harmonic mean of 126. Larvae for all populations were reared under relatively low-density conditions of 80 eggs/ 18 g CSMA larval medium (Bryant, 1969) to minimize selection for small size and/or faster developmental time that may affect longevity (Partridge \& Fowler, 1992). Populations were maintained with a discrete generation time of 21 days for a total of 24 generations. Based upon previous electrophoretic analyses in our laboratory on similar experimental lines, the minimal effective population sizes of the LARGE and SMALL lines were 500 and 50, respectively (Bryant et al., 1999). At generation 19 the 12 SMALL lines were mixed by sampling sufficient eggs to produce approximately 150 adults from each line and emerging adults were allowed to intermate freely. The MIXED population was maintained for five generations to reduce genetic disequilibrium as a consequence of the initial mixing of possibly genetically disparate lines, and then assayed for longevity at generation 24 along with the other lines (see below).

Life-history parameters were assayed at generations 0 (BASE population), at experimental generations 6, 12 and 24 for the LARGE and SMALL populations, and at generation 24 for the MIXED population. Because of the workload, only 6 of the 12 SMALL lines were assayed across the experiment. For these assays, larval cultures from all lines were set up with a standard density of 80 eggs per $18 \mathrm{~g}$ of CSMA medium (Bryant, 1969). Adults were isolated as virgins within $12 \mathrm{~h}$ of emerging and random virgin male-female pairs per line were placed into single population cages consisting of a plastic cup (with air holes) inverted over a Petri dish. All pairs were 
fed daily with dilute evaporated milk and supplied with CSMA larval medium for egg laying, to provide a complete life-history schedule for fecundity and mortality per line. Egg-to-adult viability was obtained by culturing eggs laid by females at a standard density of 80 eggs per $18 \mathrm{~g}$ of CSMA medium and counting the number of emerging adults. Emerging adults were dried and weighed as a group within each line, to obtain a mean dry weight per fly per line at each assay period. The number of pairs per line at each assay were 30 (LARGE), 20 (SMALL) and 60 (MIXED), totalling 120 pairs for the LARGE and SMALL treatments.

\section{Results}

Nearly all flies lived up to Day 21, when life span was curtailed, but thereafter, mortality increased across generations for both sexes in the LARGE and the SMALL lines (Figs 1, 2). As a result, mean survivorship decreased significantly with time under curtailed life span, for both sexes, and the drop was nearly
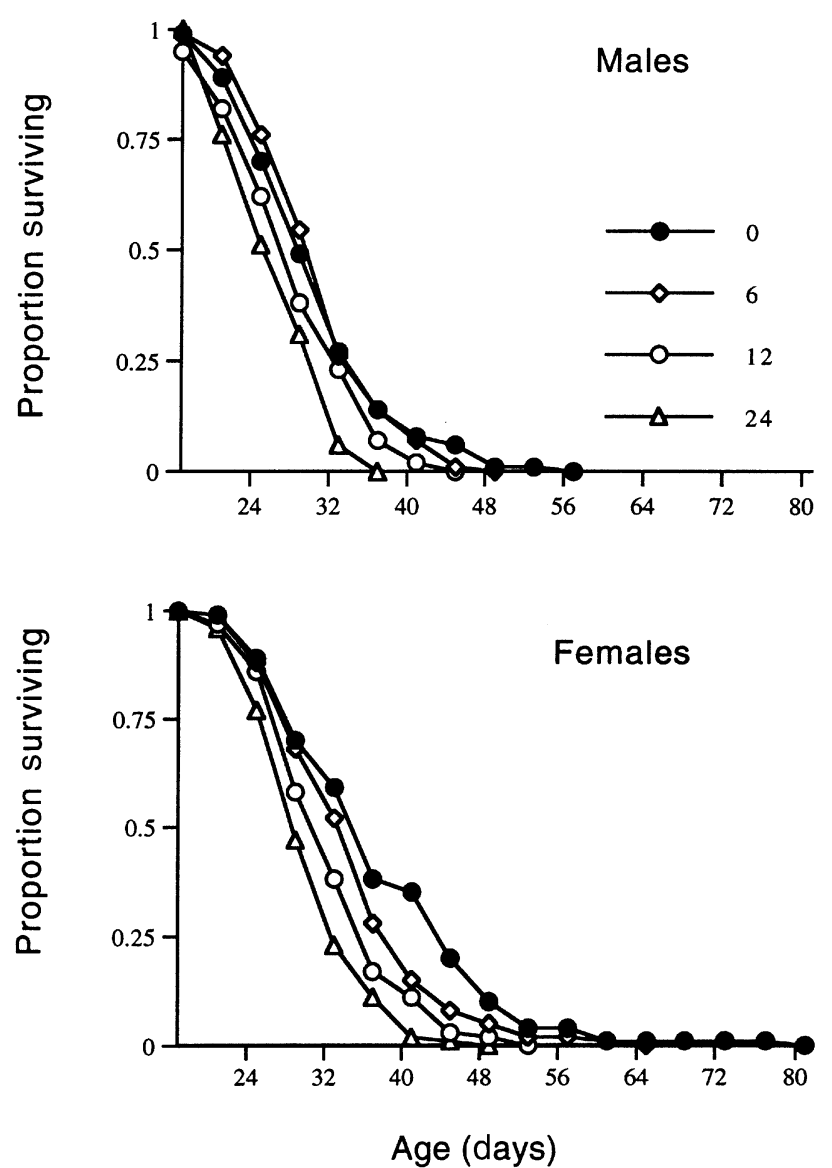

Fig. 1 Survivorship curves, measured in days from the egg stage, for females and males of the LARGE lines across the 24 generations of the experiment. identical between the two population size treatments (Fig. 3). Maximal longevity also decreased in the lines across generations, dropping from 77 days to 49 days for females and from 55 days to 36 days in males. The decrease in longevity for females was greater than that for males in both population size treatments, yielding coefficients of regression of $b=-0.24$ days per generation for females and $b=-0.15$ days per generation in males; as a result, over the course of the 24 generations females experienced an average reduction in longevity of $19 \%$ compared to only $13 \%$ for males. For both sexes the rate of reduction in longevity was slightly greater in the LARGE than in the SMALL populations $(b=-0.22$ days per generation and $b=-0.0 .17$ days per generation, respectively), suggesting that selection (including laboratory adaptation) may have played a role in these changes in longevity. However, such selectional effects were relatively minor because the rates of loss in longevity in the two treatments were not significantly different from each other.
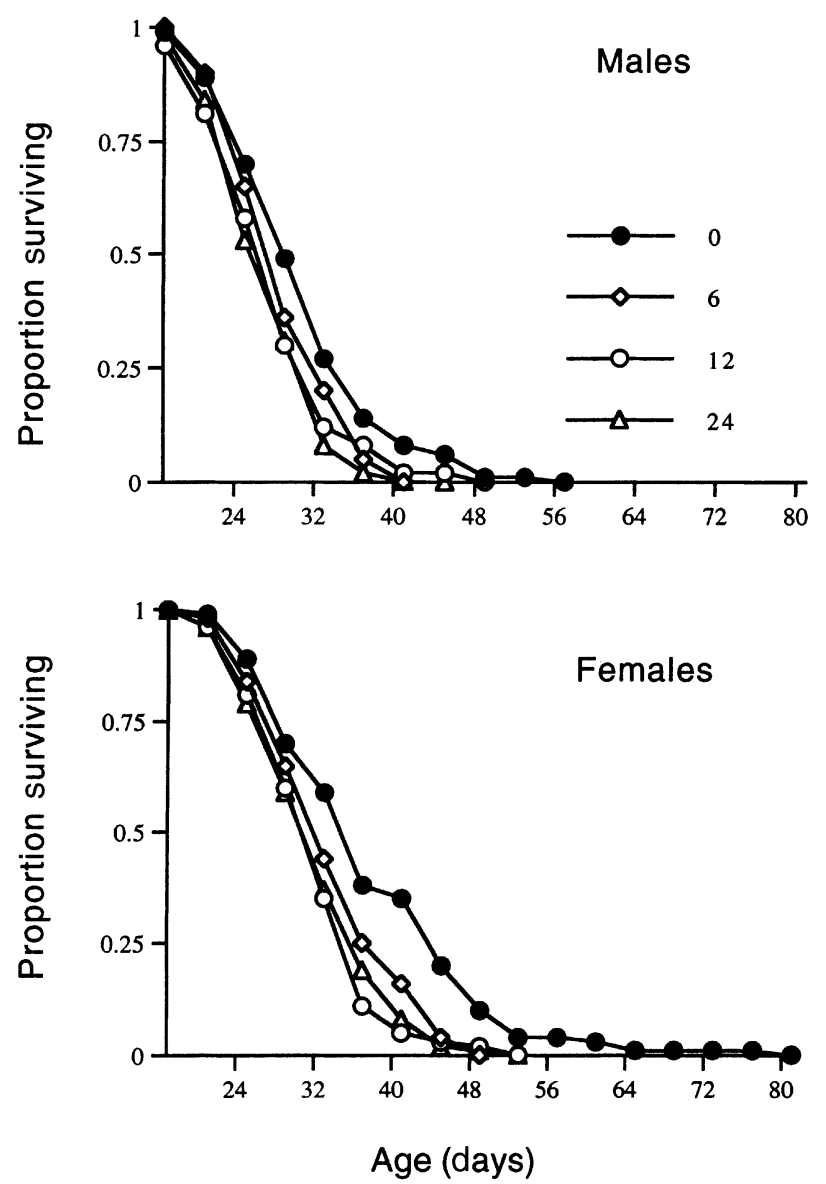

Fig. 2 Survivorship curves, measured in days from the egg stage, for females and males of the SMALL lines across the 24 generations of the experiment. 
Longevity at generation 24 in the MIXED population was nearly identical to the BASE population (generation 0 ) regardless of sex, despite longevity being significantly lower in both the LARGE and SMALL populations at this generation (Table 1). Hence, losses in longevity were restored largely by crossing the SMALL lines, suggesting that genetic effects accumulated independently across the populations. Similar results occurred for larval viability and total lifetime fecundity (Table 1); there was a significant difference between the MIXED population and both the LARGE and SMALL popu-

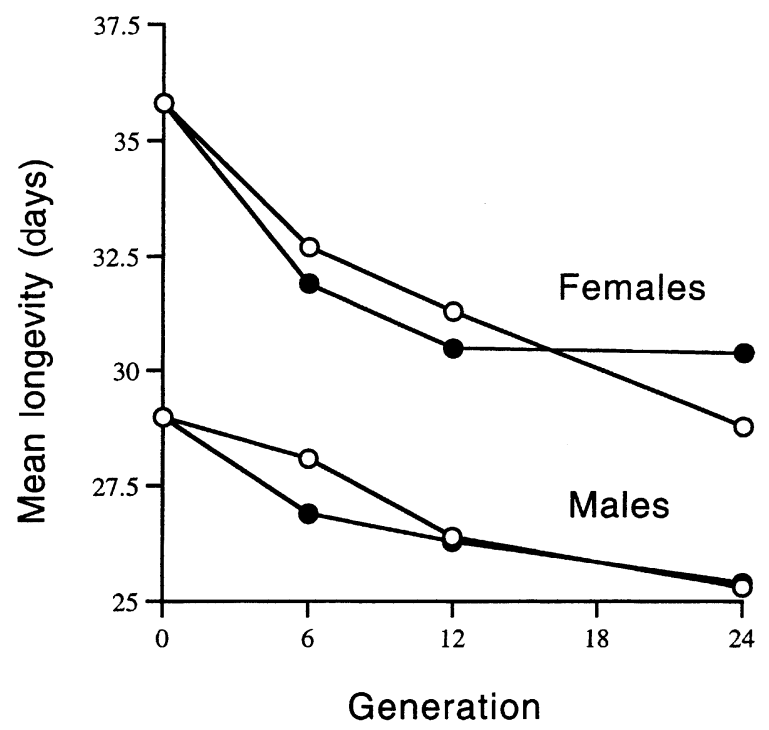

Fig. 3 Mean longevities, separately for each sex and treatment (LARGE lines, open circles, SMALL lines, closed circles) over the 24 generations of the experiment. Based on pooled amongline variances within treatments of 0.61 , the least significant difference between treatments was 1.70 ; there was a significant difference between sexes but not between treatments for any generation. lations, but not between the MIXED and BASE population (Table 1).

Longevity, fecundity and viability were all depressed in the curtailed life span populations relative to the BASE population (Table 1); the situation for larval developmental time was the opposite, however, being faster in the LARGE populations than in the BASE (developmental time was not measured in the SMALL lines). There appeared to be inadvertent selection for faster larval development in the LARGE populations, despite controlling larval density. Faster larval developmental time often affects longevity through reduced fly size (Partridge \& Fowler, 1992). This is unlikely to be the complete source of decreased longevity here because fly size changed only slightly across generations: a mean fly weight of $2.22 \mathrm{mg}$ at generation 24 was not significantly less than the mean fly weight of $2.28 \mathrm{mg}$ in the BASE population.

While longevity did not differ between the LARGE and SMALL populations, larval viability and total fecundity were lower in the SMALL than in the LARGE populations (Table 1). Both viability and total fecundity are expressed over the entire life span and subject to selection, so they would not have been entirely neutral under curtailed life span and could have been affected by inbreeding in the SMALL populations, leading to additional fitness loss due to inbreeding depression.

Total fecundity can be subdivided into contributions accruing before and after Day 21, corresponding to the date life span was curtailed. Contributions to total fecundity accruing after Day 21 under curtailed life span would be neutral, to the extent they are independent of early life fecundity, and thus losses in late-life fecundity should be equal between the population size treatments, while those for early fecundity would not. The reductions in early and late-life total fecundity were,

Table 1 Means and standard errors for longevity (in days), larval developmental time (in days), larval viability (in percent) and total fecundity (total eggs laid) for the BASE population at generation 0 and the LARGE, SMALL, and MIXED populations at generation 24

\begin{tabular}{lccccc}
\hline Population & $\begin{array}{c}\text { Female } \\
\text { longevity }\end{array}$ & $\begin{array}{c}\text { Male } \\
\text { longevity }\end{array}$ & $\begin{array}{c}\text { Developmental } \\
\text { time }\end{array}$ & $\begin{array}{c}\text { Larval } \\
\text { viability }\end{array}$ & $\begin{array}{c}\text { Total } \\
\text { fecundity }\end{array}$ \\
\hline BASE & $35.8 \pm 1.29$ & $29.0 \pm 0.91$ & $13.1 \pm 0.05$ & $79.4 \pm 1.90$ & $385 \pm 18.7$ \\
LARGE & $28.8 \pm 0.22$ & $25.3 \pm 0.18$ & $11.7 \pm 0.03$ & $70.0 \pm 2.45$ & $238 \pm 7.1$ \\
SMALL & $30.4 \pm 1.05$ & $25.4 \pm 0.62$ & - & $41.7 \pm 0.04$ & $194 \pm 17.8$ \\
MIXED & $36.6 \pm 1.69$ & $30.4 \pm 1.37$ & $13.2 \pm 0.05$ & $80.2 \pm 2.93$ & $343 \pm 24.3$ \\
$t$-tests $\dagger$ & & & & & \\
BASE vs. LARGE & $0.38 \mathrm{NS}$ & $0.85 \mathrm{NS}$ & $1.41 \mathrm{NS}$ & $0.23 \mathrm{NS}$ & $1.36 \mathrm{NS}$ \\
LARGE vs. MIXED & $4.57^{* * *}$ & $3.69^{* * *}$ & $2.57^{*}$ & $2.67^{*}$ & $3.91^{* * * *}$ \\
SMALL vs. MIXED & $3.12^{* *}$ & $3.33^{* *}$ & - & $13.04^{* * *}$ & $4.95^{* * *}$ \\
\hline
\end{tabular}

$\dagger$ Four treatments allow for three a priori tests; NS = nonsignificant at $P>0.05 ; * P<0.05 ; * * P<0.01 ; * * * P<0.005$. 
Table 2 Realized fecundity (means of total eggs laid per female and their standard errors) occurring before 21 days (early) and after 21 days (late) and the percent decrease from the BASE population

\begin{tabular}{lcccrrr}
\hline & \multicolumn{2}{c}{ Fecundity } & & \multicolumn{2}{c}{ Percentage loss } \\
\cline { 2 - 3 } Population & \multicolumn{2}{c}{ Early } & Late & & Early & Late \\
\hline BASE & $148.3 \pm 7.2$ & $236.6 \pm 25.5$ & & - & - \\
LARGE & $119.6 \pm 4.1$ & $124.5 \pm 5.1$ & & 19.4 & 47.4 \\
SMALL & $82.3 \pm 7.7$ & $111.5 \pm 24.0$ & & 44.0 & 52.9 \\
MIXED & $146.0 \pm 10.6$ & $197.0 \pm 32.7$ & & 1.5 & 16.7 \\
$t$-tests $\dagger$ & & & & & \\
BASE vs MIXED & $0.18 \mathrm{NS}$ & $0.96 \mathrm{NS}$ & & & \\
BASE vs LARGE & $2.43^{* *}$ & $4.31^{* * *}$ & & & \\
BASE vs. SMALL & $4.84^{* * *}$ & $3.57^{* * *}$ & & & \\
\hline
\end{tabular}

$\dagger \mathrm{NS}=$ nonsignificant at $P>0.05 ; * P<0.05$; ** $P<0.01$; *** $P<0.001$.

respectively, $19 \%$ and $47 \%$ for the LARGE populations and $44 \%$ and $53 \%$ for the SMALL populations. The losses in early fecundity were significantly greater in the SMALL than in the LARGE populations but were not significantly different during late life-history (Table 2). The near equality of loss in late-life fecundity (but not early life fecundity) between treatments suggests that deleterious alleles affecting late-life fecundity were accruing randomly while those affecting early life fecundity were apparently affected by inbreeding depression in the SMALL populations. Nevertheless, early life fecundity also dropped moderately $(19 \%)$ in the LARGE lines, which is not explained by either the mutation accumulation and antagonistic pleiotropy hypotheses and may be tied to the modest reduction in fly size over the course of the experiment due to shortened developmental time.

\section{Discussion}

Our results are consistent with the hypothesis that deleterious alleles affecting both late-life mortality and late-life fecundity were rendered neutral by the curtailed life span protocol and their random drift resulted in significant reductions in these life-history traits within the 24 generations of the experiment. Considering the time-frame of the experiment, decreased longevity was unlikely to be a result of new mutations but rather to mutations pre-existing in the base population that drifted to higher frequencies when selection was removed. This interpretation rests upon there being mutations with agespecific effects, as originally envisioned by Charlesworth (1990). While there is some direct evidence that mutations have age-restricted effects on longevity (Nuzhdin et al., 1997), the extent of age-specific effects of spontaneous mutations on longevity remains problematic (Houle et al., 1994; Pletcher et al., 1998; Promislow \&
Tartar, 1998). Even though mutation accumulation is consistent (and perhaps parsimonious) with our results, a number of other processes may have also contributed to declining longevity in these populations, including inbreeding depression, adaptation to the laboratory environment, systematic environmental changes in the laboratory and antagonistic pleiotropy.

Inbreeding depression does not seem a likely cause of decreased longevity in these experiments. The minimal effective population sizes of the LARGE lines of 500 would have lead to approximately $2 \%$ inbreeding over the course of 24 generations. This level of inbreeding is unlikely to account for the more extreme losses in longevity $(19 \%$ and $13 \%$ for females and males, respectively) and or fecundity (38\%) in the LARGE lines. Moreover, longevity decreased slightly more in the LARGE than in the SMALL populations (Table 1), the opposite of what is expected under inbreeding. Nevertheless, inbreeding depression may have affected early life fecundity and larval viability, as these traits were affected more in the SMALL populations than in the LARGE ones (Table 1).

Any systematic environmental effects across generations that would lower longevity, independent of accumulated genetic effects, should have also affected the MIXED populations. The near equality of longevity in the MIXED and LARGE lines argues against systematic environmental changes being a likely cause of decreasing longevity in these lines. Nevertheless, adaptation to the laboratory environment could have caused rapid changes in life-history traits through time and could have affected all lines, including the MIXED population. To minimize this effect, we allowed for an initial refractory period of 5 generations for acclimation and adaptation to the laboratory environment before beginning our experiments. Nevertheless, there was a hint of more rapid loss in mean longevity from 
generations $0-6$ than from generations 6-24, particularly in females (Fig. 3), which is not predicted under mutation accumulation (Charlesworth, 1984). Selection should have been more effective in the LARGE populations than in the SMALL populations, whereas longevity decreased nearly the same in the LARGE and SMALL lines. Moreover, if selection were responsible for decreased longevity, this should have occurred in concert across populations by affecting the same alleles in all populations, so crosses among populations should not have restored longevity. While adaptation to the laboratory environment cannot be ruled out entirely, it seems more parsimonious to assume that alleles affecting longevity were rendered neutral by curtailed life span and were subject to random drift in all populations regardless of size.

Antagonistic pleiotropy predicts a trade-off between early and late fitness, particularly for fecundity (Rose \& Charlesworth, 1980). Early and late fecundity among lines within treatments at generation 24 were positively correlated in these experiments $(r=0.65)$, as was longevity and early fecundity $(r=0.53)$ and longevity and larval viability $(r=0.67)$; thus there was no evidence of any trade-off between these life-history traits. In addition, early life fecundity did not increase in the LARGE populations, which would be expected under antagonistic pleiotropy. Inadvertent selection for faster larval development time, which apparently occurred in the LARGE populations, could confound evidence in favour of antagonistic pleiotropy, because early emerging flies may be smaller and produce smaller clutches. There was indeed a trend towards smaller flies over the course of the experiment.

On the other hand, there was a negative correlation between longevity and early progeny production, the total number of offspring per female before Day 21 $(r=-0.73, P<0.05)$. There was some evidence therefore for antagonistic pleiotropy: on average longer-lived flies produced fewer offspring early in life than shorterlived flies did. Trade-offs between shortened life span and time to sexual maturation, larval developmental time, and/or body weight have also been reported (Partridge \& Fowler, 1992; Roper et al., 1993; Zwaan et al., 1995; Nunney, 1996). Such trade-offs are not apparent in our data because: (i) truncation selection on individuals unable to mature and sexually reproduce before 21 days was extremely weak (98.5\% of 462 pairs that laid eggs, laid them on or before Day 21); (ii) there were no statistically significant changes in age at first egg laying; (iii) there were no significant changes in the time interval between the first and second clutches; and (iv) there was no significant change in mean fly size across generations. Overall, then, decreases in longevity seemed to be independent of changes in some life-history traits (fecundity, larval viability), but not in others (early progeny production).

Many base stocks are kept under discrete generation regimes, that would result in a rapid reduction in fitness and an increase in additive genetic variance with age, due to accumulation of late-acting deleterious mutations (Promislow \& Tartar, 1998). Over the 24 generations of the experiment, we observed losses of $19 \%$ and $13 \%$ for females and males, respectively. Hence, the argument of Promislow \& Tartar (1998) that many longevity experiments have been initiated with base populations that had already experienced considerable mutation accumulation, which would confound the interpretation of results, particularly with respect to antagonistic pleiotropy, has considerable merit. Nevertheless, early loss in longevity in our experiment (generations 0-6) was slightly more rapid than later losses (generations 6-24) (Fig. 3) and suggests that some other process, such as laboratory adaptation, may have confounded losses in early viability. The rate of decrease in longevity over generations 6-24 was $0.4 \%$ per generation and nearly consistent with the rate of reduction from mutation accumulation predicted by Charlesworth (1984) for viability and by Promislow \& Tartar (1998) for other fitness traits. Whatever the ultimate cause of reduced longevity, our results confirm that populations kept under discrete generation protocols may experience considerable shortening of longevity within a short period of time and, hence, may no longer represent the true longevity of these same organisms under natural conditions, as predicted by Promislow \& Tartar (1998).

\section{Acknowledgements}

Support for this study was supplied from the National Science Foundation (NSF) Dissertation Improvement Award (DEB-9520609 to D.H.R.), NSF grant (DEB90249980 to E.H.B.) and the University of Houston Coastal Center. We thank N. Barton, L. Meffert, J. Seamon, R. Sokal, J. Jacobson, J. Lester, D. Zeh and several anonymous reviewers for comments on previous drafts of the manuscript.

\section{References}

BRYANT, E. H. 1969. The fates of immatures in mixtures of two housefly strains. Ecology, 50, 1049-1069.

BRYANT, E. H., BACKUS, V. L., CLARK, M. E. AND REED, D. H. 1999. Experimental tests of captive breeding programs for endangered species. Conserv. Biol., 13, 1487-1496.

CHARLESWORTH, B. 1984. The evolutionary genetics of life histories. In: Shorrocks, B. (ed.) Evolutionary Ecology, pp. 117-133. Blackwell Scientific Publications, Oxford. 
CHARLESWORTH, B. 1990. Optimization models, quantitative genetics, and mutation. Evolution, 44, 520-538.

CHARlesworth, B. 1994. Evolution in Age-Structured Populations, 2nd edn. Cambridge University Press, Cambridge.

EDNEY, E. B. AND GILL, R. W. 1968. Evolution of senescence and specific longevity. Nature, 220, 281-282.

HAMiLton, w. D. 1966. The moulding of senescence by natural selection. J. Theor. Biol., 12, 12-45.

HOUle, D., HUGHES, K. A., HOFFMASTER, D. K., IHARA, J., ASSIMACOPOUlOS, S., CANADA, D. ET AL. 1994. The effects of spontaneous mutation on quantitative traits. I. Variances and covariances of life history traits. Genetics, 138, 773-785.

KIMURA, M. 1983. The Neutral Theory of Molecular Evolution. Cambridge University Press, New York.

LYNCH, M., BLANCHARD, J., HOULE, D., KIBOTA, T., SCHULTZ, S., VASSILIEVA, L. ET AL. 1999. Perspective: Spontaneous deleterious mutation. Evolution, 53, 645-663.

MedawAR, P. B. 1952. An Unsolved Problem in Biology. H.K. Lewis, London.

MUELLER, L. D. 1987. Evolution of accelerated senescence in laboratory populations of Drosophila. Proc. Natl. Acad. Sci. U.S.A., 84, 1974-1977.

NUNNEY, L. 1996. The response to selection for fast larval development in Drosophila melanogaster and its effect on adult weight: an example of a fitness trade-off. Evolution, 50, 1193-1204.

NUZHDIN, S. V., PASYUKOVA, E. G., DILDA, C. L., ZENG, Z.-B. AND MACKAY, T. F. C. 1997. Sex-specific quantitative trait loci affecting longevity in Drosophila melanogaster. Proc. Natl. Acad. Sci. U.S.A., 94, 9734-9739.

PARTRIDGE, L. AND BARTON, N. H. 1993. Optimality, mutation and the evolution of ageing. Nature, 362, 305-311.

PARTRIDGE, L. AND FOWLER, K. 1992. Direct and correlated responses to selection on age at reproduction in Drosophila melanogaster. Evolution, 46, 76-91.

Pletcher, S. D., HOUle, D. AND CuRTSinger, J. w. 1998. Agespecific properties of spontaneous mutations affecting mortality in Drosophila melanogaster. Genetics, 148, 287-303.

Pletcher, S. D., HOUSE, D. AND CURTSINGER, J. W. 1999. The evolution of age-specific mortality rates in Drosophila melanogaster: genetic divergence among unselected lines. Genetics, 153, 813-823.

PROMISLOW, D. E. L. AND TARTAR. M. 1998. Mutation and senescence: where genetics and demography meet. Genetica, 102/103, 299-314.

ROPER, C., PIGNATELlli, P. AND PARTRIDGE, L. 1993. Evolutionary effects of selection on age at reproduction in larval and adult Drosophila melanogaster. Evolution, 47, 445-455.

ROSALES, A. L., KRAFSUR, E. S. AND KIM, Y. 1994. Cryobiology of the face fly and housefly (Diptera: Muscidae). J. Med. Entomol, 31, 671-680.

ROSE, M. R. 1984. Laboratory evolution of postponed senescence in Drosophila melanogaster. Evolution, 38, 1004-1010.

ROSE, M. R. 1991. Evolutionary Biology of Aging. Oxford University Press, Oxford.

ROSE, M. R. AND CHARLESWORTH, B. 1980. A test of evolutionary theories of senescence. Nature, 287, 141-142.

SERVICE, P. M., HUTCHINSON, E. W., MACKINLEY, M. D. AND ROSE, M. R. 1985. Resistance to environmental stress in Drosophila melanogaster selected for postponed senescence. Physiol. Zool., 58, 380-389.

SOKAL, R. 1970. Senescence and genetic load: Evidence from Tribolium. Science, 167, 1733-1734.

TUCIC, N., Stojkovic, I., MILANOVIC, D. AND SESLIJA, D. 1997. Laboratory evolution of life-history traits in the bean weevil (Acanthoscelides obtectus): the effects of density-dependent and age-specific selection. Evolution, 51, 1896-1909.

WILliaMs, G. C. 1957. Pleiotropy, natural selection, and the evolution of senescence. Evolution, 11, 398-411.

ZWAAN, B. J., BIJLISMA, R. AND HOEKSTRA, R. F. 1995. On the developmental theory of ageing. I. Starvation resistance and longevity in Drosophila melanogaster in relation to pre-adult breeding conditions. Heredity, 66, 29-39. 